\title{
Testing The Casual Relation Between Sunspots And Temperature Using Wavelets Analysis
}

\author{
Abdullah Almasri \\ Lund University, Sweden \\ Ghazi Shukur \\ Jönköping and Växjö Universities
}

Follow this and additional works at: http://digitalcommons.wayne.edu/jmasm

Part of the Applied Statistics Commons, Social and Behavioral Sciences Commons, and the Statistical Theory Commons

\section{Recommended Citation}

Almasri, Abdullah and Shukur, Ghazi (2005) "Testing The Casual Relation Between Sunspots And Temperature Using Wavelets Analysis," Journal of Modern Applied Statistical Methods: Vol. 4 : Iss. 1 , Article 14.

DOI: $10.22237 /$ jmasm/1114906440

Available at: http://digitalcommons.wayne.edu/jmasm/vol4/iss1/14 


\section{Testing The Casual Relation Between Sunspots And Temperature Using Wavelets Analysis}

\author{
Abdullah Almasri \\ Department of Statistics \\ Lund University, Sweden
}

\author{
Ghazi Shukur \\ Departments of Economics and Statistics \\ Jönköping University and Växjö University
}

Investigated and tested in this article are the causal nexus between sunspots and temperature by using statistical methodology and causality tests. Because this kind of relationship cannot be properly captured in the short run (daily, monthly or yearly data), the relationship is investigated in the long run using a very low frequency Wavelets-based decomposed data such as D8 (128 - 256 months). Results indicate that during the period 1854-1989, the causality nexus between these two series is as expected of onedirectional form, i.e., from sunspots to temperature.

Key words: Wavelets, time scale, causality tests, sunspots, temperature

\section{Introduction}

The Sun is the energy source that powers Earth's weather and climate, and therefore it is natural to ask whether changes in the Sun could have caused past climate variations and might cause future changes. At some level the answer must be yes. Recently, concerns about human-induced global warming have focused attention on just how much climatic change the Sun could produce. Accordingly, many authors tried to investigate the relation between the sunspots and the climate change, e.g., Friis-Christensen (1997) compared observations of cloud cover and cosmic particles and concluded that variation in global cloud cover was correlated with the cosmic ray flux from 1980 to 1995 . They proposed the observed variation in cloud

Abdullah Almasri also holds an appointment in the Department of Primary Care and General Practice, University of Birmingham, UK. Email: abdullah.almasri@stat.lu.se. Ghazi Shukur holds appointments in the Departments of Economics and Statistics, Jönköping University and Växjö University, Sweden. cover seemed to be caused by the varying solar activity related cosmic ray flux and postulated that an accompanying change in the earth's albedo could explain the observed correlations between solar activity and climate. However, Jorgensen and Hansen (2000) showed that any evidence supporting that the mechanism of cosmic rays affecting the cloud cover and hence climate does not exist.

Nevertheless, most of these studies suffered from the lack of statistical methodology. In this study, well selected statistical tools are used to investigate the causal relation between the sunspots and the temperature. A vector autoregressive (VAR) model is constructed and applied, which allows for causality test, on low frequency Wavelets based decomposed data. Processing in this manner we can see the nature of the causal relation between these two variables.

Wavelet is a fairly new approach in analysing data (e.g. Daubechies, 1992) that is becoming increasingly popular for a wide range of applications (e.g. time series analyses). This subject is not really familiar in other areas such as in statistics with environmental application. The idea behind using this technique is based on the fact that the time period (time scale) of the analysis is very crucial for determining those aspects that are relatively more important, and those that are relatively less important. In time series one can envisage a cascade of time scales 
within which different levels of information are available. Some information is with long horizons, others with short horizons.

In this article, the discrete wavelet transform (DWT) is used in studying the relationship between the sunspots and temperature in Northern Hemisphere 1854-1989 (see Figures 1 and 2). The DWT has several appealing qualities that make it a useful method for time series, exhibiting features that vary both in time and frequency. By using the DWT, it is possible to investigate the role of time scale in sunspots and temperature relationships.

The article is organized as follows: After the introduction, the wavelets analysis is introduced. Next presented is the methodology and testing procedure used in this study. Estimated results follow, and finally, summary and the conclusion.

\section{Methodology}

The wavelet transform has been expressed by Daubechies (1992) as "a tool that cuts up data or functions into different frequency components, and then studies each component with a resolution matched to its scale." Thus, with wavelet transform, series with heterogeneous (unlike Fourier transform) or homogeneous information at each scale may be analyzed. Unlike the Fourier transform, which uses only sins and cosines as basis functions, the wavelet transform can use a variety of basis functions.

The wavelet decomposition is made with respect to the so-called Symmlets basis. Thus, a brief presentation about this decomposition methodology, which called the discrete wavelete transform (DWT), is given.

$$
\text { Let } \mathbf{X}=\left(X_{0}, X_{2}, \ldots, X_{T-1}\right)^{\prime} \text { be a }
$$

column vector containing $T$ observations of a real-valued time series, and assume that $\mathrm{T}$ is an integer multiple of $2^{M}$, where $M$ is a positive integer. The discrete wavelet transform of level $J$ is an orthonormal transform of $\mathbf{X}$ defined by

$$
\mathbf{d}=\left(\mathbf{d}_{1}, \mathbf{d}_{2}, \ldots, \mathbf{d}_{j}, \ldots, \mathbf{d}_{J}, \mathbf{s}_{J}\right)^{\prime}=\mathbf{W X},
$$

where $\mathbf{W}$ is an orthonormal $T \times T$ real-valued matrix, i.e. $\quad \mathbf{W}^{-1}=\mathbf{W}^{\prime} \quad$ so $\mathbf{W}^{\prime} \mathbf{W}=\mathbf{W W}^{\prime}=\mathbf{I}_{T} . \quad \mathbf{d}_{j}=\left\{d_{j, k}\right\}$, $j=1,2, \ldots, J$, are $T / 2^{j} \times 1$ real-valued vectors of wavelet coefficients at scale $j$ and location $k$.

The real-valued vector $\mathbf{s}_{J}$ is made up of $T / 2^{J}$ scaling coefficients. Thus, the first $T-T / 2^{J}$ elements of $\mathbf{d}$ are wavelet coefficients and the last $T / 2^{J}$ elements are scaling coefficients, where $J \leq M$. Notice that the length of $\mathbf{X}$ does coincide with the length of $\mathbf{d}$ (length of $\mathbf{d}_{j}=2^{M-j}$, and $\mathbf{s}_{J}=2^{M-J}$ ).

The multiresolution analysis of the data leads to a better understanding of wavelets. The idea behind multiresolution analysis is to express $\mathbf{W}^{\prime} \mathbf{d}$ as the sum of several new series, each of which is related to variations in $\mathbf{X}$ at a certain scale. Because the matrix $\mathbf{W}$ is orthonormal, the time series may be constructed from the wavelet coefficients $\mathbf{d}$ by using $\mathbf{X}=\mathbf{W}^{\prime} \mathbf{d}$.

Partition the columns of $\mathbf{W}^{\prime}$ commensurate with the partitioning of $\mathbf{d}$ to obtain

$$
\mathbf{W}^{\prime}=\left[\begin{array}{lllll}
\mathbf{W}_{1} & \mathbf{W}_{2} & \ldots & \mathbf{W}_{J} & \mathbf{V}_{J}
\end{array}\right],
$$

where $\mathbf{W}_{j}$ is a $T \times T / 2^{j}$ matrix and $\mathbf{V}_{J}$ is a $T \times T / 2^{J}$ matrix. Define the multiresolution analysis of a series by expressing $\mathbf{W}^{\prime} \mathbf{d}$ as a sum of several new series, each of which is related to variations in $\mathbf{X}$ at a certain scale:

$$
\mathbf{X}=\mathbf{W}^{\prime} \mathbf{d}=\sum_{j=1}^{J} \mathbf{W}_{j} \mathbf{d}_{j}+\mathbf{V}_{J} \mathbf{s}_{J}=\sum_{j=1}^{J} \mathbf{D}_{j}+\mathbf{S}_{J}
$$


Figure 1: Monthly data of the sunspots.

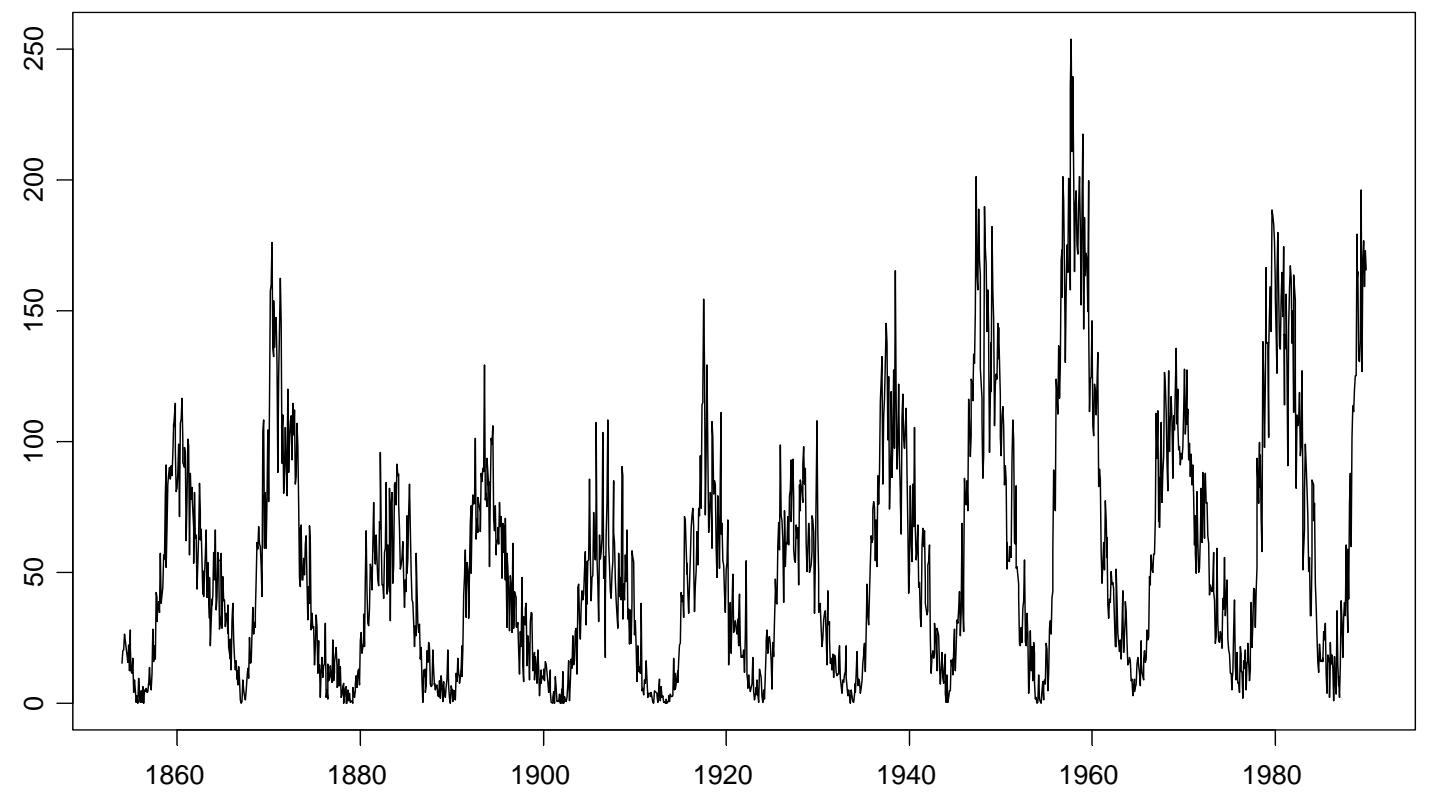

Figure 2: Monthly data of the Northern Hemisphere temperature.

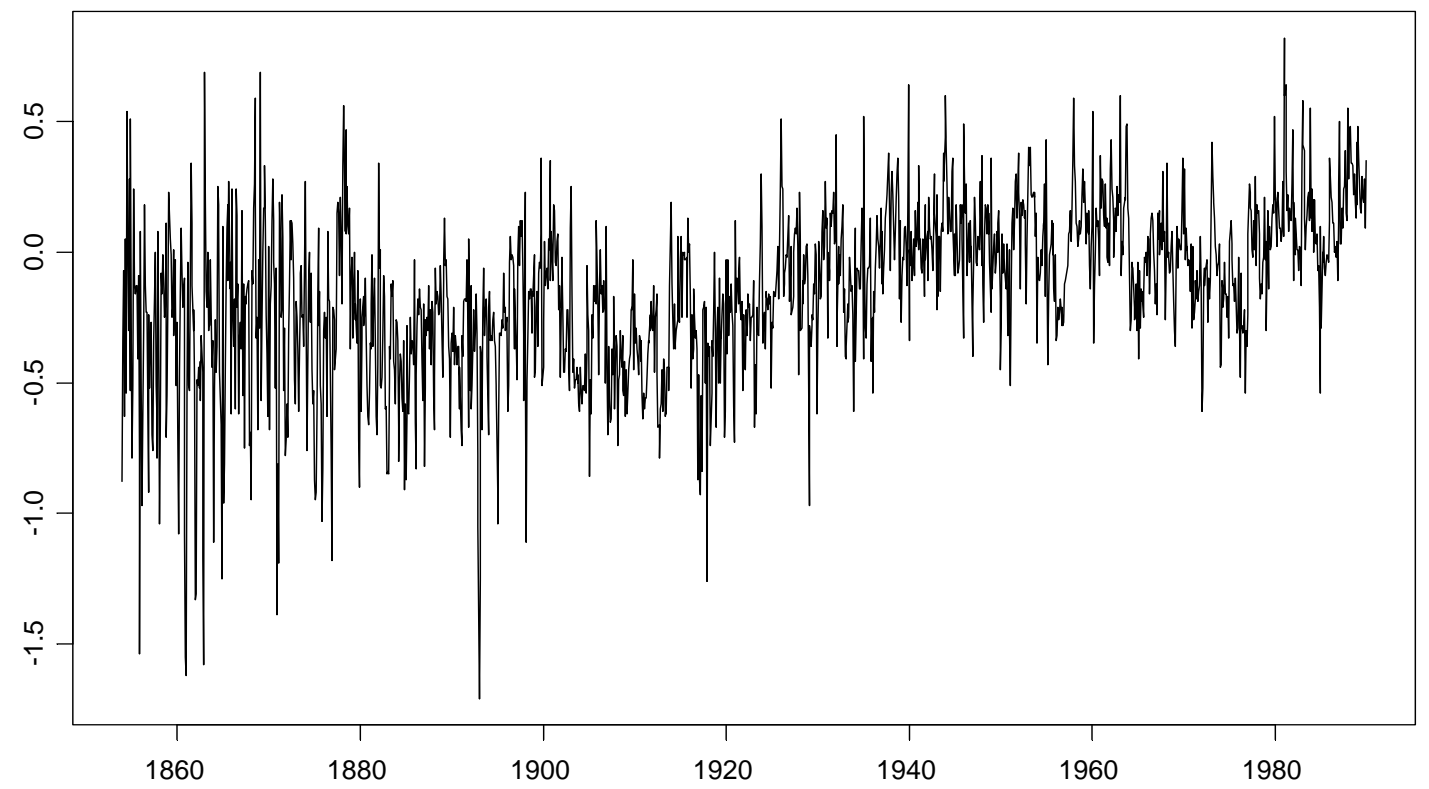

Monthly temperature for the northern hemisphere for the years 1854-1989, from the data base held at the Climate Research Unit of the University of East Anglia, Norwich, England (Briffa. \& Jones, 1992). The numbers consist of the temperature (degrees C) difference from the monthly average over the period 1950-1979. 
The terms in the previous equation constitute a decomposition of $\mathbf{X}$ into orthogonal series components $\mathbf{D}_{j}$ (detail) and $\mathbf{S}_{J}$ (smooth) at different scales, and the length of $\mathbf{D}_{j}$ and $\mathbf{S}_{J}$ coincides with the length of $\mathbf{X}(T \times 1$ vector $)$. Because the terms at different scales represent components of $\mathbf{X}$ at different resolutions, the approximation is called a multiresolution decomposition, see Percival and Mofjeld (1997).

As mentioned earlier the wavelet decompositions in this paper will be made with respect to the Symmlets basis. This has been done by using the S-plus Wavelets package produced by StatSci of MathSoft that was written by Bruce and Gao (1996). Figure 3 shows the multiresolution analysis of order $J=6$ based on the Symmlets of length 8 .

The Causality Between the Sunspots and Temperature

Next the wavelets analysis is used in investigating the hypothesis that the sunspots may affect the temperature. This will mainly be done by using causality test. Because this kind of relationship can not properly be captured in the short run (daily, monthly or yearly data), only the relationship in the long run is investigated, either by using 10-20 years data (which is not available in this case) or a very low frequency Wavelets decomposed data like D8 (128 - 256 months). This is used in this article (see Figure 3). This will be done empirically by constructing a (VAR) model that allows for causality test in the Granger sense.

Causality is intended as in the sense of Granger (1969). That is, to know if one variable precedes the other variable or if they are contemporaneous. The Granger approach to the question whether sunspots (Sun) causes temperature (Tem) is to see how much of the current value of the second variables can be explained by past values of the first variable. (Tem) is said to be Granger-caused by (Sun) if (Sun) helps in the prediction of (Tem), or equivalently, if the coefficients of the lagged (Sun) are statistically significant in a regression of (Tem) on (Sun). Empirically, one can test for causality in Granger sense by means of the following vector autoregressive (VAR) model:

$$
\begin{gathered}
\text { Tem }_{t}=a_{0}+\sum_{i=1}^{k} a_{i} \text { Tem }_{t-i}+\sum_{i=1}^{k} b_{i} \text { Sun }_{t-i}+e_{1 t}, \\
\text { Sun }_{t}=c_{0}+\sum_{i=1}^{k} c_{i} \text { Tem }_{t-i}+\sum_{i=1}^{k} f_{i} \text { Sun }_{t-i}+e_{2 t},
\end{gathered}
$$

where $e_{1 t}$ and $e_{2 t}$ are error terms, which are assumed to be independent white noise with zero mean. The number of lags, $k$, will be decided by using the Schwarz (1978) information criteria, in what follows referred to as SC.

According to Granger and Newbold (1986) causality can be tested for in the following way: A joint $F$-tests is constructed for the inclusion of lagged values of (Sun) in (1) and for the lagged values of (Tem) in (2). The null hypothesis for each $F$-test is that the added coefficients are zero and therefore the lagged (Sun) does not reduce the variance of (Tem) forecasts (i.e. $b_{i}$ in (1) are jointly zero for all $i$ ), or that lagged (Tem) does not reduce the variance of (Sun) forecasts (i.e. $f_{i}$ in (2) are jointly zero for all $i$ ). If neither null hypothesis is rejected, the results are considered as inconclusive.

However, if both of the $F$-tests rejected the null hypothesis, the result is labeled as a feedback mechanism. A unique direction of causality can only be indicated when one of the pair of $F$-tests rejects and the other accepts the null hypothesis which should be the case in the study.

Moreover, before testing for causality, the augmented Dickey-Fuller $(1979,1981)$ is applied, in what follows referred to as ADF, test for deciding the integration order of each aggregate variable. When looking at the Wavelets decomposed data for sun and temperature used here, i.e. the D8 in Figure 3 below, the ADF test results indicate that each variable is integrated of the same order zero, i.e., $\mathrm{I}(0)$, indicating the both of the series are stationary implying that the VAR model can be estimated by standard statistical tools. 
Figure 3: The solid line denotes the D8 for the sunspots data and the dotted line denotes the D8 for the temperature.

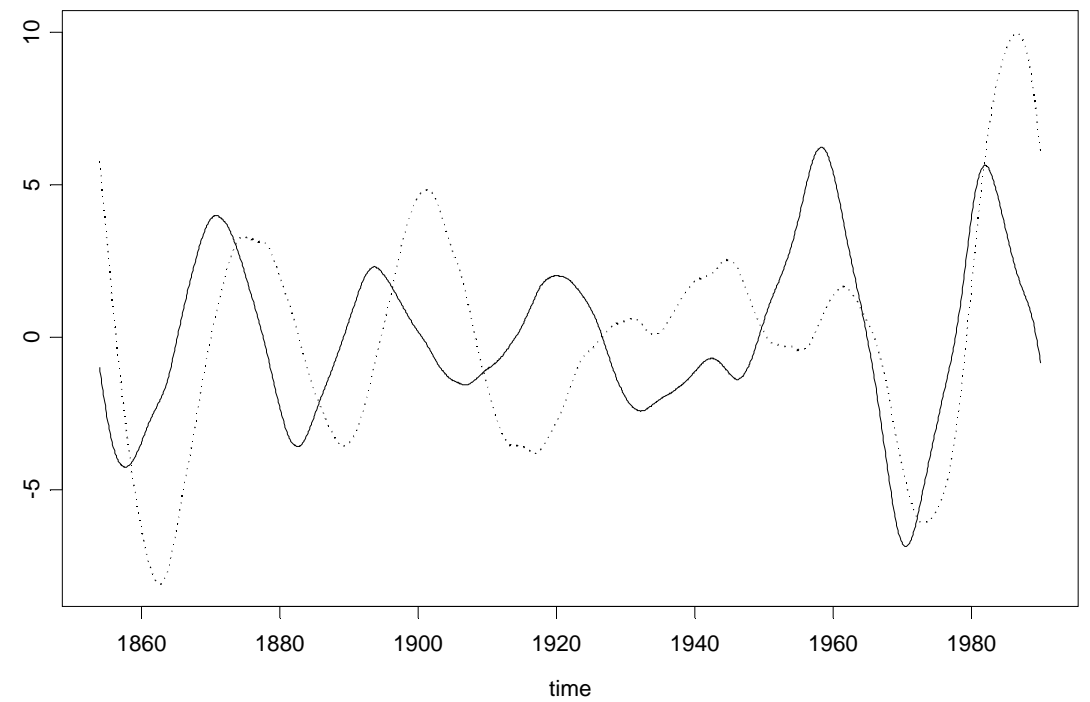

Results

According to the model selection criteria proposed by Schwarz (1978), it is found that the model that minimizes this criteria is the VAR(3). When this model is used to test for causality, the inference is drawn that only the (Sun) Granger causes the (Tem). The test results can be found in Table 1, below. This means that the causality nexus between these two series is a onedirectional form, i.e., from (Sun) to (Tem). This should be fairly reasonable, since it is not logical to assume that the temperature in the earth should have any significant effect on the sunspots.

Table 1: Testing results for the Granger causality.

\begin{tabular}{cc} 
Null Hypothesis & P-value \\
\hline Sun does not Granger Cause Tem & 0.0037 \\
Tem does not Granger Cause Sun & 0.5077
\end{tabular}

\section{Conclusion}

The main purpose of this article is to model the causality relationship between sunspots and temperature. Although other studies exist for the similar purpose, they are not based on a careful statistical modeling. Moreover, these studies have sometimes shown to end up with conflicting results and inferences. Here, in this article, well selected statistical methodology for estimation and testing the causality relation between these two variables is used.

A very low frequency Wavelets based decomposed data indicates that, during the period 1854-1989, the causality nexus between these two series is the expected one-directional form, i.e., from sunspots to temperature

\section{References}

Bruce, A. G., \& Gao, H.-Y. (1996). Applied wavelet analysis through S-Plus, New York: Springer-Verlag. 
Daubechies, I. (1992). Ten lectures on wavelets, Volume 61 of CBMS-NFS Regional Conference Series in Applied Mathematics. Philadelphia: Society for Industrial and Applied Mathematics.

Dickey, D. A., \& Fuller, W. A. (1979). Distribution of the estimators for autoregressive time series with a unit root. Journal of the American Statistical Association, 74, 427-431.

Dickey, D. A., \& Fuller, W. A. (1981). The likelihood ratio statistics for autoregressive time series with a unit root. Econometrica, 49, 1057-1072.

Granger, C. W. J., \& Newbold, P. (1986). Forecasting economic time series, $2^{\text {nd }}$ New York: Academic Press.
Granger, C. W. J. (1969). Investigating causal relations by econometric models an crossspectral methods. Econometrica, 37, 24-36.

Percival, D. B., \& Mofjeld, H. O. (1997). Analysis of subtidal coastal sea level fluctuations using Wavelets. Journal of the American Statistical Association, 92(439), 868880.

Percival, D. B., \& Walden, A. T. (2000). Wavelet methods for time series analysis. Cambridge, UK: Cambridge University Press.

Schwarz, G. (1978). Estimation the dimention of a model. Annals of Statistics, 6, 461-464. 